\title{
Fuzzy Prediction Interval Models for Forecasting Renewable Resources and Loads in Microgrids
}

\author{
Doris Sáez, Senior Member, IEEE, Fernanda Ávila, Daniel Olivares, Member, IEEE, \\ Claudio Cañizares, Fellow, IEEE, and Luis Marín
}

\begin{abstract}
An energy management system (EMS) determines the dispatching of generation units based on an optimizer that requires the forecasting of both renewable resources and loads. The forecasting system discussed in this paper includes a representation of the uncertainties associated with renewable resources and loads. The proposed modeling generates fuzzy prediction interval models that incorporate an uncertainty representation of future predictions. The model is demonstrated using solar and wind generation and local load data from a real microgrid in Huatacondo, Chile, for one-day ahead forecasts to obtain the expected values together with fuzzy prediction intervals to represent future measurement bounds with a certain coverage probability. The proposed prediction interval models would help to enable the development of robust microgrid EMS.
\end{abstract}

Index Terms-Energy management system (EMS), forecasting, fuzzy modeling, microgrid, prediction intervals, renewable.

\section{INTRODUCTION}

$\mathbf{T}$ HE INTEGRATION of intermittent energy sources such as wind and solar energy in power systems brings significant new challenges to the operation and planning of these systems. Traditional dispatch models can no longer guarantee a reliable operation of the system due to high levels of uncertainty associated with intermittent power sources, and new dispatch techniques need to be developed [1], [2]; this issue takes even more relevance in the operation of microgrids. The presence of energy storage systems (ESSs) can help reduce the effects of uncertainty, facilitating the integration of variable sources if a properly designed energy management system (EMS) is in place; however, the performance of such an EMS will depend on the accuracy of the employed forecasting system and the manner in which uncertainty is accounted for in the EMS.

Manuscript received January 2, 2014; revised May 20, 2014, August 20, 2014, and November 12, 2014; accepted November 16, 2014. Date of publication December 19, 2014; date of current version February 16, 2015. This work was supported in part by the Millennium Institute Complex Engineering Systems under Grant ICM: P-05-004-F and Grant CONICYT: FBO16; in part by National Fund for Science and Technology under Project 1140775; and in part by CONICYT/FONDAP/15110019. Paper no. TSG-00005-2014

D. Sáez, F. Ávila, and L. Marín are with the Department of Electrical Engineering, University of Chile, Santiago 6513027, Chile (e-mail: dsaez@ing.uchile.cl).

D. Olivares is with the Department of Electrical Engineering, Pontificia Universidad Católica de Chile, Santiago 7820436, Chile.

C. Cañizares is with the Department of Electrical and Computer Engineering, University of Waterloo, Waterloo, ON N2L-3G1, Canada.

Color versions of one or more of the figures in this paper are available online at http://ieeexplore.ieee.org.

Digital Object Identifier 10.1109/TSG.2014.2377178
The aforementioned issues become even more challenging in the context of stand-alone microgrids, where the limited number of generators and significant levels of intermittent source penetration require a highly coordinated operation of the controllable assets. Thus, in stand-alone microgrids, load, and wind and solar power profiles are less smooth, and present more high-frequency changes than those observed in bulk power systems, due to limited geographical area covered by these grids. As a result, the demand-supply balance is typically more critical.

To address the high levels of uncertainty associated with the intermittency of resources, new dispatch schemes that use stochastic formulations of the dispatch problem are an alternative to achieve the desired levels of reliability [1], [3], [4]. These formulations require forecasting systems that provide not only the expected future resources but also a measure of their variability, such as prediction intervals or representative scenarios. Consequently, conventional forecasting methodologies are not completely suitable for microgrid applications, making the development of new techniques necessary.

To solve the load forecasting problem, computational intelligence techniques, such as neural networks [5], [6], have been widely used. Palma-Behnke et al. [7] discussed a neural network for two-days ahead electric consumption forecasting for an EMS. Additionally, other techniques, such as wavelet analysis, have also been used as a complementary tool, for example, to characterize different load profiles [8]. Evolutionary algorithms are also used for determining the inputs (e.g., type of day, temperature) to load-predictive models [9], and a new bi-level strategy for short-term load forecasting is presented in [10], and demonstrated using real data from a university campus in Canada. However, these load forecasting methods do not explicitly consider the modeling of forecast uncertainty.

Existing literature on probabilistic and interval forecasting has focused on long-term analysis for investment and planning purposes, which do not require the forecasts to be represented as time-series. For instance, McSharry et al. [11] estimated the expected value of future demand, as well its prediction density, considering explanatory variables such as temperature and seasonality. Hyndman and Fan [12] presented long-term density forecasts for demand using temperature as an explanatory variable. Hong et al. [13] proposed interval prediction for long-term demand using similar input variables based on scenarios. These techniques are not readily applicable to short-term forecast, which is the main focus of this paper, 
where values in consecutive time steps are strongly coupled and density forecast is not relevant.

The forecasting of renewable sources in microgrids depends on the accuracy of the forecast models with respect to weather conditions (e.g., wind speed and direction, solar radiation, temperature). For forecasting wind and solar power generation, there are two main approaches: 1) to forecast the wind speed or solar radiation and convert it to power using an empirical formula (see [14], [15]) and 2) is to directly forecasts the power generation (see [16], [17]).

Most models of both solar and wind resources and power do not directly represent uncertainty. Hence, as the main contribution of this paper, fuzzy prediction interval models, which provide a systematic framework for the representation of uncertainty and thus are suitable for forecasting loads and renewable-based energy generation in microgrids are developed. A somewhat similar approach was recently reported in [18], where a short-term load forecast with prediction intervals for bulk power systems is presented, based on fuzzy sets for the model parameters; however, this paper differs from [18] in that intervals are calculated based on the covariance of data for various operating points, which are characterized by linear regression models with fixed parameters as discussed in detail later.

The rest of this paper is organized as follows. Section II provides details on the potential applications of this paper in the context of isolated microgrids. In Section III, a brief description of fuzzy modeling and the derivation of fuzzy interval models and fuzzy prediction intervals are provided, including their representation. Section IV presents forecasting results based on fuzzy prediction interval modeling for wind power, solar power, and load of an actual microgrid located in Chile. Section $\mathrm{V}$ provides the main conclusion and contributions of this paper.

\section{Forecasting ApPliCATIONS In MiCROgRids}

The EMS of microgrids dispatches distributed generators based on a unit commitment, so that certain objectives are achieved. There are two main approaches for the EMS architecture: 1) centralized and 2) distributed. Olivares et al. [19] argued that a centralized EMS is more suitable for remote microgrids operating in islanded mode, where the goal is to provide continuous operation of the system under variable generation and load. A centralized EMS, illustrated in Fig. 1, determines the dispatching of units (and control commands for controllable loads) by solving an optimization problem. For this purpose, the EMS is provided with relevant information for each generation unit and load in the microgrid (e.g., cost functions, technical characteristics/limitations, network parameters, and modes of operation), as well as information from forecasting systems (e.g., local load, wind speed, and solar irradiance), to determine the dispatching of resources according to the selected objectives. The estimation of future parameter values requires forecasting system with a certain coverage probability (CP). Uncertainty in forecasting load, wind speed, and solar irradiance is typically indirectly accounted for by using a model predictive control (MPC) approach for dispatch

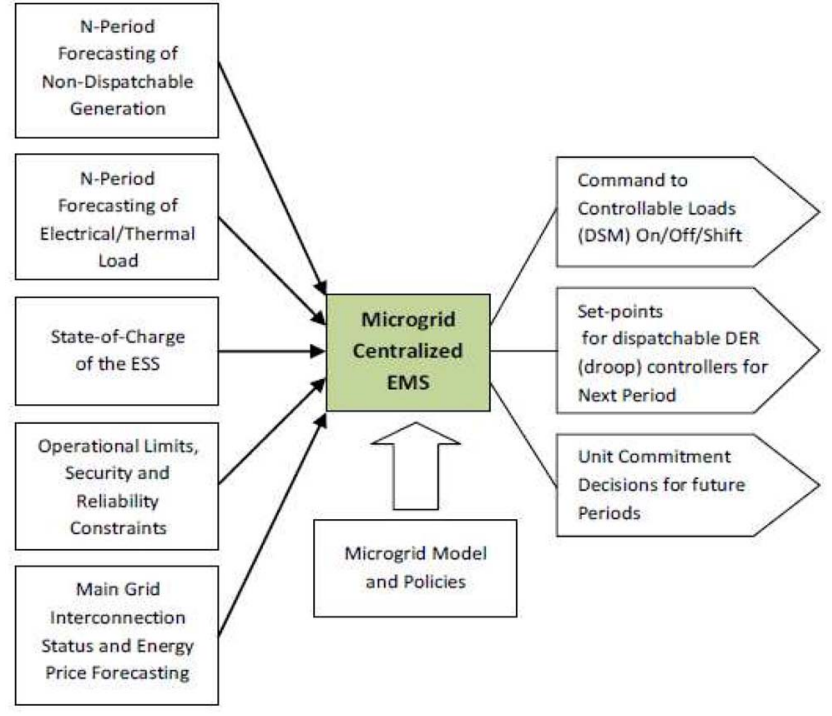

Fig. 1. Centralized EMS for a microgrid.

in the EMS [20]. In this approach, a deterministic multistep optimization problem is solved iteratively over time using the most updated/accurate information available at each time step [21], [22]. However, this approach may not be able to provide the desired levels of reliability in dispatch operations, as it does not directly account for uncertainty. Hence, new approaches using stochastic EMS formulations are needed to address this issue. These stochastic formulations would enable a determination of the necessary reserves of the microgrid thus avoiding the need to arbitrarily fix these reserves a-priory.

The robust optimization approach combines the worst-case analysis with a min-max formulation to obtain optimal solutions that are robust against variations in the parameter values with respect to a nominal value (optimal worst-case scenario). A particular formulation of the robust energy management problem, similar to the formulation presented in [3] is as follows:

$$
\begin{aligned}
& \min _{u_{k_{t}}} \max _{p_{k_{t}}} \sum_{k_{t}} F\left(y_{k_{t}}, z_{k_{t}}, u_{k_{t}}, p_{k_{t}}\right) \\
& \text { s.t. } z_{k_{t}+1}=w\left(y_{k_{t}}, z_{k_{t}}, u_{k_{t}}, p_{k_{t}}\right) \quad \forall k_{t} \\
& g_{k_{t}}\left(y_{k_{t}}, z_{k_{t}}, u_{k_{t}}, p_{k_{t}}\right)=0 \quad \forall k_{t} \\
& h_{k_{t}}\left(y_{k_{t}}, z_{k_{t}}, u_{k_{t}}, p_{k_{t}}\right) \leq 0 \quad \forall k_{t} \\
& \left\|u_{k_{t}+1}-u_{k_{t}}\right\| \leq \Delta u_{k_{t}}^{\max } \quad \forall k_{t} \\
& p_{k_{t}}=\hat{p}_{k_{t}}+\overline{s_{k t}} \Delta \hat{p}_{k t}-\underline{s_{k t}} \Delta \hat{p}_{k t} \quad \forall k_{t} \\
& \sum_{k_{t}}\left(\overline{s_{k t}}+\underline{s_{k t}}\right) \leq \Gamma
\end{aligned}
$$

where $z_{k_{t}} \in R^{w}$ is a vector of discrete time-dependent variables, such as the state-of-charge of storage systems; $\hat{p}_{k_{t}} \in R^{l}$ is the vector of parameters representing the best available estimation at step $k_{t}=1$ of for example, system demand, intermittent generation, and fuel prices, for all of the time steps in the multistage horizon; and $\Delta \hat{p}_{k t} \in R^{l}$ is the vector of maximum variations of parameter realization $p_{k_{t}} \in R^{l}$ with respect to the estimated $\hat{p}_{k_{t}} \in R^{l}$. Vector $u_{k_{t}} \in R^{n}$ represents 
the control variables, and vector $y_{k_{t}} \in R^{n}$ represents timeindependent variables, such as voltages, phase angles, and frequency. Vectors $\overline{s_{k t}} s_{k t} \in\{0,1\}$ are binary variables that control the direction of variation of $p_{k_{t}}$ with respect to the predicted $\hat{p}_{k_{t}}$, and finally the parameter $\Gamma$, called the budget of uncertainty, is the limit on the number of timesteps in the prediction that are allowed to deviate from the forecasted value. Nonlinear functions $w: R \rightarrow R^{W}, g: R \rightarrow R^{g}$, $h: R \rightarrow R^{h}$ correspond to, for example, power-flow equations and ESS balance equations. The set of inequality constraints guarantees that control variables do not change by more than a maximum acceptable amount between two consecutive time steps.

The value of the parameter $\Delta \hat{p}_{k_{t}}$ is usually estimated as a percentage of $\hat{p}_{k_{t}}$ and adjusted depending on the accuracy of the forecasting method and forecasting horizon.

In the past, fuzzy models have been used in the forecasting of power outputs from nondispatchable units, due to their suitability as approximators of nonlinear dynamic processes. In this paper, a forecasting method using a new type of fuzzy prediction interval models is derived based on [23] and [24], which not only provides the forecasted output but also their prediction intervals, which can be readily used to obtain the parameter $\Delta \hat{p}_{k_{t}}$ in the robust energy management problem formulation.

\section{FuZzy PREDiction InTERVAL MOdELING}

\section{A. Takagi and Sugeno Fuzzy Modeling}

Takagi and Sugeno (T\&S) [25] described a type of fuzzy model that is suitable for approximating a large class of nonlinear systems. The premises are based on fuzzy sets, and the consequences are linear models that represent different operating points of the system. The T\&S fuzzy models can be represented by the following expression:

$$
\begin{aligned}
& \hat{p}_{t}=f^{\mathrm{TS}}\left(x_{t-1}^{p}, p_{t-1}, u_{t-1}\right) \\
& \hat{p}_{t}=\sum_{j=1}^{m} \beta_{j}\left(x_{t-1}^{p}\right) p_{t}\left(p_{t-1}, u_{t-1}\right)
\end{aligned}
$$

where $t$ is time, $x_{t-1}^{p}$ is the vector of premises; $p_{t-1}$ is the past output vector; $u_{t-1}$ is the past control vector; $\beta_{j}$ is the degree of activation for rule $R_{j}$; and $p_{t j}$ is the output of the local model for rule $j\left(R_{j}\right)$. In matrix form, the T\&S model is represented as

$$
\begin{aligned}
\hat{p}_{t} & =\Phi^{T} \theta \\
\Phi_{j}^{T} & =\beta_{j}\left(x_{t-1}^{p}\right)\left[1 p_{t-1} u_{t-1}\right] \\
\Phi^{T} & =\left[\Phi_{1}^{T}, \ldots, \Phi_{m}^{T}\right] \\
p_{t j}\left(p_{t-1}, u_{t-1}\right) & =\left[1 p_{t-1} u_{t-1}\right] \theta_{j}=\psi^{T} \theta_{j} \\
\theta^{T} & =\left[\theta_{1}, \ldots, \theta_{m}\right]
\end{aligned}
$$

where $\Phi^{\boldsymbol{T}}$ is the fuzzy matrix regression and $\boldsymbol{\theta}$ is the parameter matrix. The set of data samples can be written as follows:

$$
p_{i}=\psi^{T}\left(m_{i}\right) \theta+e_{i}
$$

where $m_{i}$ are the measurement input variables for $i=1, \ldots, N$, and $e_{i}$ are the forecasting errors.

In this paper, the method in [26] was considered, which minimizes the number of rules making a partition of the space of output variables universe that is projected into the input space finding the optimal fuzzy sets and rules. This partition is based on a fuzzy clustering method, and allows obtaining the premise parameters. The consequence parameters are obtained using the T\&S method based on least squares, as described in [27].

\section{B. Fuzzy Interval Modeling}

Interval modeling is a method by which one can approximate function families that are given by a finite set of input and output measurements. The interval defines a band that contains the measurement values with a certain CP.

Škrjanc [23] presented an analytical derivation of fuzzy intervals, which results in lower and upper fuzzy models with a defined CP. Thus, the expected covariance of the residuals between the observed data and the local model output is given as follows:

$$
\Delta \hat{p}_{j}=\operatorname{cov}\left(p_{j}-\hat{p}_{j}\right)=\widehat{\sigma}_{j}^{2} I+\widehat{\sigma}_{j}^{2} \psi_{j}^{T}\left(\psi_{j} \psi_{j}^{T}\right)^{-1} \psi_{j}
$$

where $p_{j}$ is the output of the local model $j$ and the variance of the local noise signal is $E\left\{e_{j} e_{j}^{T}\right\}=\widehat{\sigma}_{j}^{2} \boldsymbol{I}$. For a set of data samples, the lower and upper bounds of the local linear models $j$ are defined, respectively, as

$$
\begin{aligned}
& \underline{f}_{j}\left(m_{i}\right)=\psi_{i, j}^{T} \theta_{j}-\underline{\alpha} \Delta \hat{p}_{i, j} \\
& \bar{f}_{j}\left(m_{i}\right)=\psi_{i, j}^{T} \theta_{j}+\bar{\alpha} \Delta \hat{p}_{i, j}
\end{aligned}
$$

where

$$
\Delta \hat{p}_{i, j}=\widehat{\sigma}_{j}\left(1+\psi_{i, j}^{T}\left(\psi_{j} \psi_{j}^{T}\right)^{-1} \psi_{i, j}\right)^{1 / 2}
$$

for $i=1, \ldots, N$. Here, $\bar{\alpha}$ and $\underline{\alpha}$ are the parameters of the fuzzy intervals that should be tuned. Note that narrower bands imply a greater number of data points outside the bands. Using (17)-(19) and applying the T\&S framework, the lower and upper bounds for the output are represented by

$$
\begin{aligned}
& \underline{\hat{p}_{t}}=f^{\mathrm{TS}}\left(x_{t-1}^{p}, p_{t-1}, u_{t-1}\right)-\underline{\alpha}_{t} I^{\mathrm{TS}}\left(x_{t-1}^{p}, p_{t-1}, u_{t-1}\right) \\
& \overline{\hat{p}_{t}}=f^{\mathrm{TS}}\left(x_{t-1}^{p}, p_{t-1}, u_{t-1}\right)+\overline{\alpha_{t}} I^{\mathrm{TS}}\left(x_{t-1}^{p}, p_{t-1}, u_{t-1}\right)
\end{aligned}
$$

with the fuzzy interval given by

$$
I^{\mathrm{TS}}\left(x_{t-1}^{p}, p_{t-1}, u_{t-1}\right)=\sum_{j=1}^{m} \beta_{j}\left(x_{t-1}^{p}\right) \Delta \hat{p}_{t j}
$$

where $\alpha_{t}$ and $\overline{\alpha_{t}}$ are time-variant parameters related to the interval width and are tuned using experimental data to obtain a certain $\mathrm{CP}$. The parameters of fuzzy interval models correspond to the fuzzy regression matrix $\Phi_{j}^{T}$ obtained from the fuzzy model identification (premise parameters) and the variance of the local noise signal $\widehat{\sigma}_{j}^{2}$.

The CP is defined as

$$
C P=\frac{\sum_{i=1}^{M} \delta_{i}}{M}
$$


where $M$ is the number of the data set and $\delta_{i}$ are binary variables that indicate whether the sample data $m_{i}$ belong to the interval, i.e., $\underline{\hat{p}_{t}} \leq m_{i} \leq \overline{\hat{p}}_{t}$.

\section{Fuzzy Prediction Intervals}

This paper proposes the use of fuzzy prediction interval models for forecasting the output of future steps ahead. In this case, (8) and (9) can be reformulated as follows:

$$
\begin{gathered}
\hat{p}_{k_{t}}=f^{\mathrm{TS}}\left(x_{k_{t}-1}^{p}, p_{k_{t}-1}, u_{k_{t}-1}\right) \quad \forall k_{t}=t, \ldots, t+K \\
\hat{p}_{k_{t}}=\sum_{j=1}^{m} \beta_{j}\left(x_{k_{t}-1}^{p}\right) p_{k_{t}}\left(p_{k_{t}-1}, u_{k_{t}-1}\right) \\
\quad \forall k_{t}=t, \ldots, t+K .
\end{gathered}
$$

The prediction of the lower and upper prediction intervals at future steps ahead is considered as a function of the output until the previous step, real, and/or forecasted depending on the number of steps ahead, as follows:

$$
\begin{gathered}
\underline{\hat{p}_{k_{t}}}=f^{\mathrm{TS}}\left(x_{k_{t}-1}^{p}, p_{k_{t}-1}, u_{k_{t}-1}\right)-\alpha_{k_{t}} I^{\mathrm{TS}}\left(x_{k_{t}-1}^{p}, p_{k_{t}-1}, u_{k_{t}-1}\right) \\
\overline{\hat{p}_{k_{t}}}=f^{\mathrm{TS}}\left(x_{k_{t}-1}^{p}, p_{k_{t}-1}, u_{k_{t}-1}\right)+\alpha_{k_{t}} I^{\mathrm{TS}}\left(x_{k_{t}-1}^{p}, p_{k_{t}-1}, u_{k_{t}-1}\right) \\
\quad \forall k_{t}=t, \ldots, t+K
\end{gathered}
$$

where the vector $\alpha_{k_{t}}$ is chosen to be the same for both the upper and lower intervals. The tuning of the vector $\alpha_{k_{t}}$ is obtained using the experimental data and (23) to calculate a certain CP. First, an initial value of $\alpha_{k_{t}}$ is chosen, and with this value, the interval forecast is performed for the required steps ahead. Next, with these results, the CP is calculated using (23). If this is less than expected, the value of $\alpha_{k_{t}}$ is increased, and the procedure is repeated until the required $\mathrm{CP}$ is achieved.

\section{Case Study}

\section{A. Huatacondo Microgrid}

In this paper, the load and the wind and solar energy resources of a small, isolated microgrid, in the village of Huatacondo (20 $\left.50^{\circ} \quad 36.37^{\prime \prime} \quad \mathrm{S} \quad 69^{\circ} 3^{\prime} \quad 8.71^{\prime \prime} \quad \mathrm{W}\right)$ in the Atacama Desert, Chile, were considered. The microgrid is composed of two photovoltaic systems $\left(P_{\max }^{S}=\right.$ $24 \mathrm{~kW})$; a wind turbine $\left(P_{\max }^{w}=5 \mathrm{~kW}\right)$; the existing diesel generator unit in the village which is typical of isolated grids, an ESS composed of a lead-acid battery bank connected to the grid through a bidirectional inverter; a water pump; and loads $\left(L_{\max }=28 \mathrm{~kW}\right)$. Fig. 2 illustrates the various components of this microgrid, which features an EMS that minimizes the operational costs while supplying the demanded load, considering a one-day ahead forecasting of the wind and solar resources and the electrical load [20].

The forecasting results are based on fuzzy prediction interval modeling for the wind power, solar power, and load, which are required by the EMS. Given the particular application considered in this paper, i.e., microgrids for remote communities,

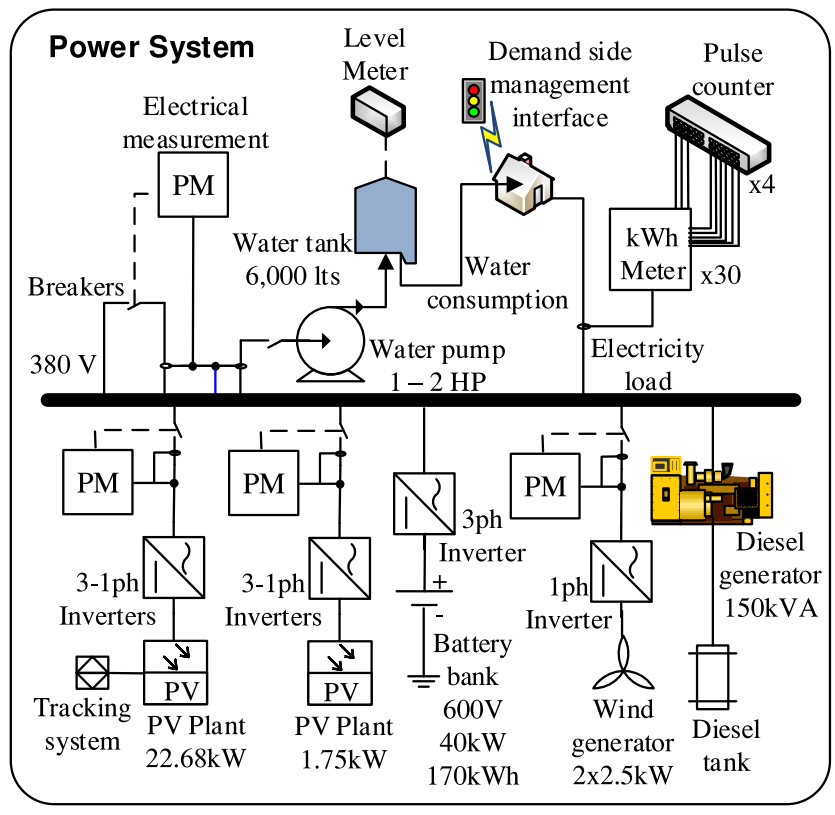

Fig. 2. Schematics of a renewable-based microgrid.

the simplicity of the forecasting method is a desirable trait. For this reason, a forecasting system that is able to provide reasonable predictions based only on a reduced set of variables that are easy and inexpensive to acquire, such as the power output is used here. Other explanatory variables such as wind speed and temperatures may help improve the forecast, but are expensive and difficult to obtain in remote communities such as Huatacondo; furthermore, the impact of some of these variable are in some way "embedded" in the power output information. It is also worth mentioning that for the particular remote community used in this paper, the load is much more dependent on factors such as TV-schedules, breakfast, lunch, and dinner times, and school schedules than on short-term environmental factors [28]. Hence, only solar and wind generation output powers and load demand at previous selected time-steps are considered as inputs to the forecasting system.

The results presented were obtained for a forecast horizon of $24 \mathrm{~h}$ and a sampling time of $15 \mathrm{~min}$, which results in 96 steps in one-day. Note that the periods of time used for training and validation of the models differ for wind, solar, and load powers, given that no reliable data was available from all of them for the exact same period of time, due to technical problems with the data acquisition systems at Huatacondo.

\section{B. Forecasting of Wind Power}

For fuzzy modeling of the wind power, training data were obtained from July 27 to August 28, 2010, and validation data were obtained from August 29 to September 26, 2010. The wind power $\left(p=P^{W}\right)$ model was derived based on the measurements of the previous day, with a sampling time of $15 \mathrm{~min}$ as follows:

$$
\hat{p}_{t}=\hat{P}_{t}^{w}=f^{\mathrm{TS}}\left(P_{t-1}^{w}, P_{t-2}^{w}, \ldots, P_{t-96}^{w}\right) .
$$




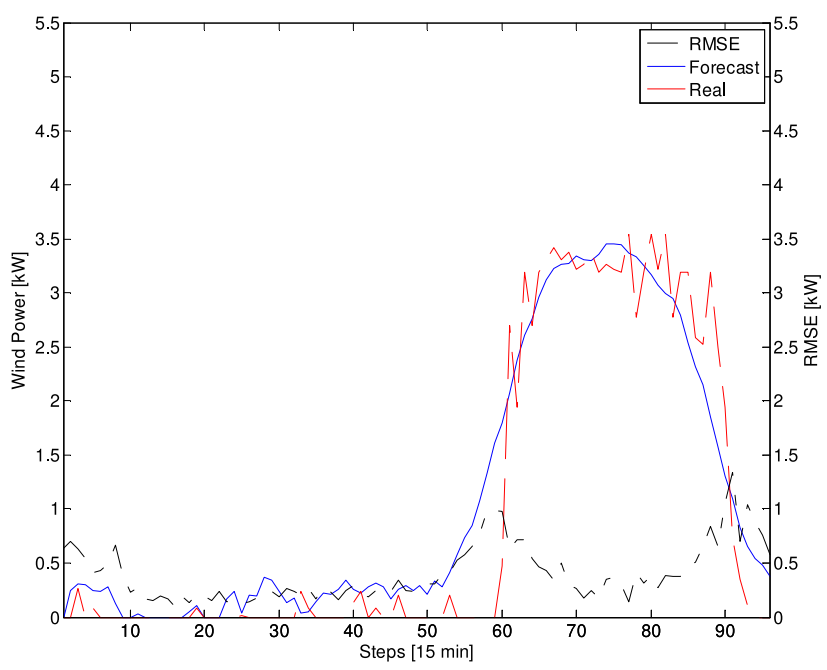

Fig. 3. One-day wind power forecasting for a given day.

Then, using the identification procedure explained in Section III, results in the following:

$$
\begin{aligned}
& R_{j} \text { if } P_{t-1}^{w} \text { is } A_{j}^{1} \text { and } P_{t-2}^{w} \text { is } A_{j}^{2} \text { and } \ldots \text { and } P_{t-96}^{w} \text { is } A_{j}^{96} \\
& \text { then } \hat{P}_{t j}^{w}=\theta_{0 j}+\theta_{1 j} P_{t-1}^{w}+\theta_{2 j} P_{t-2}^{w}+\cdots+\theta_{96 j} P_{t-96}^{w} \\
& \hat{P}_{t}^{w}=\sum_{j=1}^{m} \beta_{j}\left(x_{t-1}^{p}\right) \hat{P}_{t j}^{w}
\end{aligned}
$$

where $R_{j}$ is the $j$ rule, $A_{j}^{1}, \ldots, A_{j}^{96}$ are the fuzzy sets defined based on the clustering technique mentioned in Section III. Finally, using (22), the fuzzy prediction interval model for wind power is given by the following expression:

$$
\begin{aligned}
& R_{j} \text { if } P_{t-1}^{w} \text { is } A_{j}^{1} \text { and } P_{t-2}^{w} \text { is } A_{j}^{2} \text { and } \ldots P_{t-96}^{w} \text { is } A_{j}^{96} \\
& \quad \text { then } \triangle \hat{P}_{t j}^{w} \\
& \triangle \hat{P}_{t}^{w}=\sum_{j=1}^{m} \beta_{j}\left(x_{t-1}^{p}\right) \triangle \hat{P}_{t j}^{w} .
\end{aligned}
$$

Fig. 3 shows the wind power forecast using one specific known day starting at midnight. Additionally, this figure presents the root mean square error (RMSE) for 1 to 96-step ahead forecast for a set of six days, showing that the peaks of the RMSE occur when the power suddenly increases or decreases.

Fig. 4 presents the fuzzy prediction intervals for different coverage probabilities (associated with the parameter $\alpha$ ) for one-day ahead forecasting, considering the same day used in the forecast shown in Fig. 3. Note that, in general, the narrower bands cover fewer data points, while the wider bands can cover almost all of the data. For higher levels of wind power generation, wider intervals imply more uncertainty (see Fig. 4, steps 57-90). The interval with a $90 \%$ of CP is significantly wider than the other intervals because of the measures that are close to zero, which add more uncertainty to the entire model.

\section{Forecasting of Solar Power}

For fuzzy modeling of the solar power, training data corresponds to November 1, 2012, to January 16, 2013,

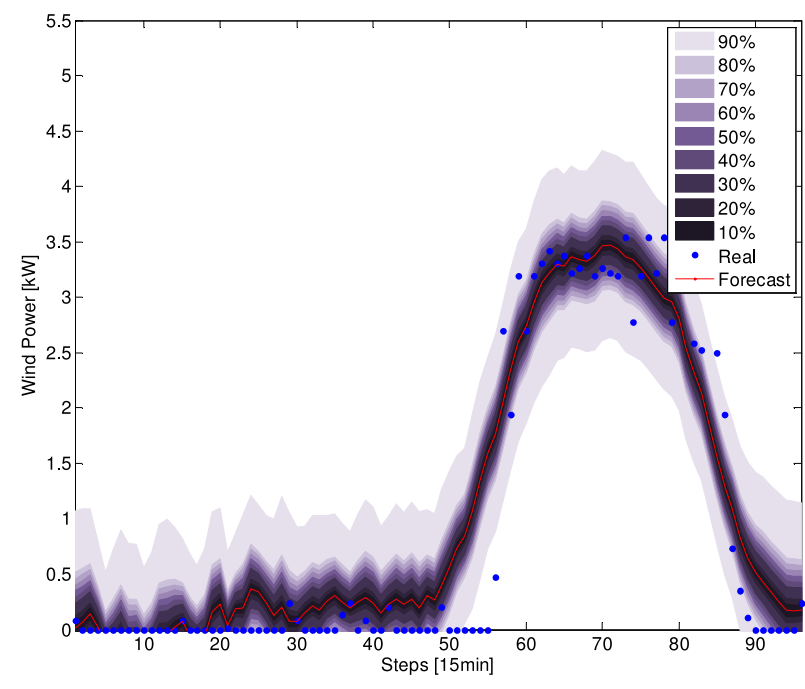

Fig. 4. Fuzzy intervals for one-day wind power forecasting for different $\mathrm{CP}$ levels, for a given day and for various values of $\alpha$.

and the validation set consists of data from January 17 to January 31,2013 . The following solar power model $\left(p=P^{S}\right)$ is assumed with a sampling time of $15 \mathrm{~min}$ :

$$
\hat{p}_{t}=\hat{P}_{t}^{s}=f^{\mathrm{TS}}\left(P_{t-1}^{s}, \ldots, P_{t-96}^{s}\right) .
$$

Thus, using the identification procedure described in Section III-A, the resulting fuzzy model for the solar power is given by

$$
\begin{aligned}
& R_{j} \text { if } P_{t-1}^{s} \text { is } A_{j}^{1} \text { and } \ldots \text { and } P_{t-96}^{s} \text { is } A_{j}^{96} \\
& \text { then } \hat{P}_{t j}^{s}=\theta_{0 j}+\theta_{1 j} P_{t-1}^{s}+\cdots+\theta_{96 j} P_{t-96}^{s} \\
& \hat{P}_{t}^{s}=\sum_{j=1}^{m} \beta_{j}\left(x_{t-1}^{p}\right) \hat{P}_{t j}^{s}
\end{aligned}
$$

where $m=2$ is the number of the fuzzy rules.

The fuzzy prediction interval model is given by the following expression:

$$
\begin{aligned}
& R_{j} \text { if } P_{t-1}^{s} \text { is } A_{j}^{1} \text { and } \ldots \text { and } P_{t-96}^{s} \text { is } A_{j}^{96} \\
& \quad \text { then } \triangle \hat{P}_{t j}^{s} \\
& \Delta \hat{P}_{t}^{s}=\sum_{j=1}^{m} \beta_{j}\left(x_{t-1}^{p}\right) \triangle \hat{P}_{t j}^{s} .
\end{aligned}
$$

Fig. 5 shows solar power forecast results for a given day. This forecast starts at midnight; therefore, null values exist at the beginning and end, and the maximum occurs near noon; this behavior is captured by the fuzzy model. In addition, the RMSE is shown for steps 1-96 forecast, considering 15 days. Similar to the wind power graph, the peaks of the RMSE also indicate when the sun power increases and decreases, representing dawn and dusk, respectively.

Fig. 6 shows the fuzzy intervals for different $\mathrm{CP}$ levels, from $10 \%$ to $90 \%$ for a one-day forecast. Observe that the intervals are narrower than for wind power, for each $\mathrm{CP}$, since a regular pattern of the sunlight is observed at this location. 


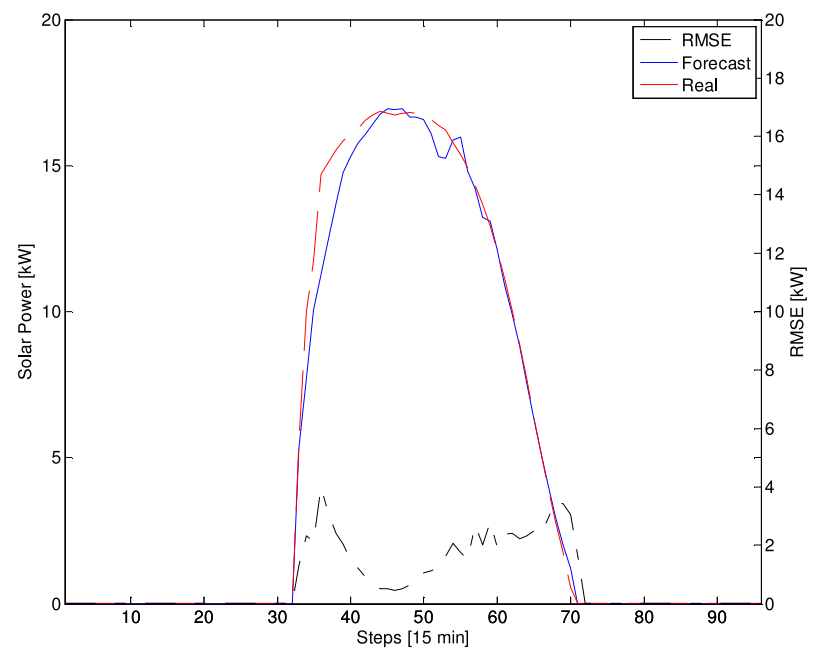

Fig. 5. One-day solar power forecasting for a given day.

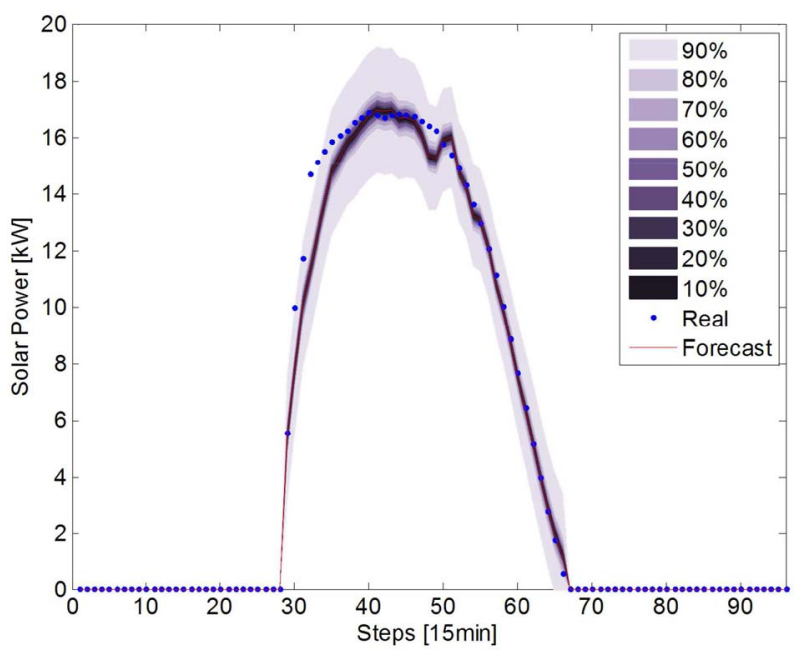

Fig. 6. Solar power fuzzy intervals for different CP levels for a one-day forecast, for a given day and for various values of $\alpha$.

\section{Load Forecasting}

For fuzzy modeling of the load, training data were obtained from November 1, 2012, to January 16, 2013, and the validation set consists of data from January 17 to January 31, 2013. Thus, the structure of fuzzy model for the load, with a sampling time of $15 \mathrm{~min}$ is given by

$$
\hat{p}_{t}=\hat{P}_{t}^{L}=f^{\mathrm{TS}}\left(P_{t-1}^{L}, \ldots, P_{t-96}^{L}\right) .
$$

Then, the predictive fuzzy model and the corresponding fuzzy prediction interval models are developed. Using the identification procedure described in Section III-A, the resulting fuzzy model for the load is given by four rules with the following form:

$$
\begin{aligned}
& R_{j} \text { if } P_{t-1}^{L} \text { is } A_{j}^{1} \text { and } \ldots \text { and } P_{t-96}^{L} \text { is } A_{j}^{96} \\
& \text { then } \hat{P}_{t j}^{L}=\theta_{0 j}+\theta_{1 j} P_{t-1}^{L}+\ldots+\theta_{96 j} P_{t-96}^{L} \\
& \hat{P}_{t}^{L}=\sum_{j=1}^{m} \beta_{j}\left(x_{t-1}^{p}\right) \hat{P}_{t j}^{L}
\end{aligned}
$$

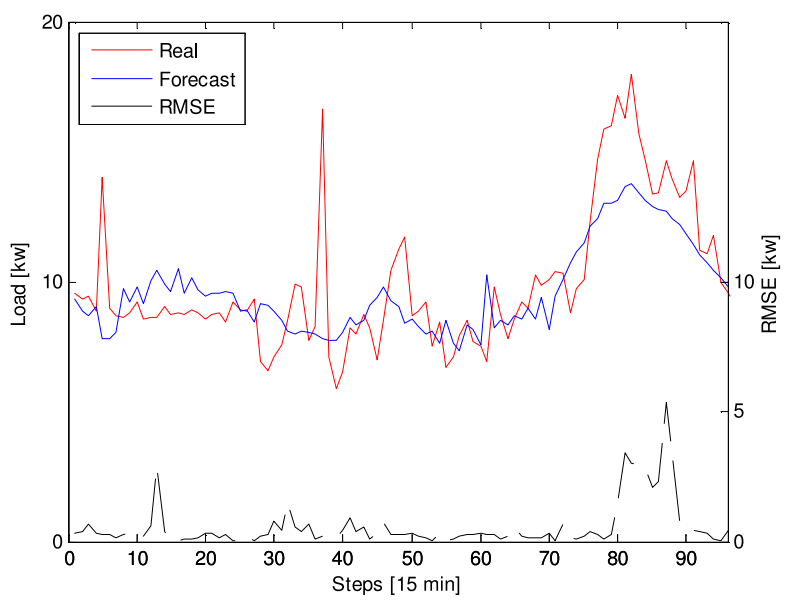

Fig. 7. Load forecasting for one-day ahead forecasting for a given day.

where $m=4$ is the number of the fuzzy rules. Hence, the fuzzy prediction interval model for the load is given by the following expression:

$$
\begin{aligned}
& R_{j} \text { if } P_{t-1}^{L} \text { is } A_{j}^{1} \text { and } \ldots \text { and } P_{t-96}^{L} \text { is } A_{j}^{96} \\
& \quad \text { then } \triangle \hat{P}_{t j}^{L} \\
& \triangle \hat{P}_{t}^{L}=\sum_{j=1}^{m} \beta_{j}\left(x_{t-1}^{p}\right) \triangle \hat{P}_{t j}^{L} .
\end{aligned}
$$

Fig. 7 shows the load forecasting from 1 to 96-steps ahead, starting at midnight using the historical data from the previous day at selected time instants, defined by the inputs of the model. A high variability of the load is observed for this small village (Huatacondo), confirming that load forecasting is an important challenge for microgrids. Additionally, this figure shows the RMSE for 1-96-steps ahead forecasts using a set of 15 days, and the peaks along the prediction steps are a result of the uncertain demand. Observe that high frequency variations are captured with less accuracy for longer look-ahead windows. However, given the MPC approach used by most EMSs, as explained in Section II, this is not an issue.

Fig. 8 shows the fuzzy intervals for different coverage probabilities for one-day ahead forecasting. In contrast with the intervals for solar and wind power, the load intervals have almost the same width throughout the entire forecast period, which means that uncertainty remains relatively constant during the entire day.

\section{E. Discussion}

A common characteristic of most EMS architectures is the use of an MPC type of control; thus, at each time step when the EMS calculations are triggered, a new forecast is performed by the forecasting system in order to use the most updated information. Hence, the EMS will use predictions for one-step ahead to 96-steps ahead for a 24-h horizon in its calculations. Therefore, the EMS will face different levels of uncertainty for predictions at different steps ahead produced by only one run of the forecasting system, with higher levels of uncertainty for the final hours of the horizon, as expected. 


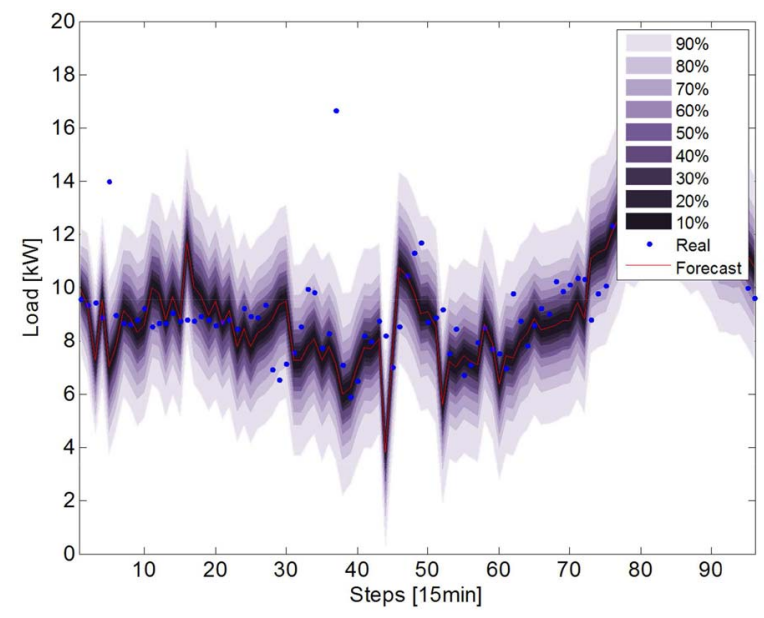

Fig. 8. One-day ahead fuzzy intervals for the load at different CP levels, for a given day and for various values of $\alpha$.

TABLE I

FORECASTING ERRORS

\begin{tabular}{crr}
\hline \hline & $\begin{array}{r}\text { One-step ahead } \\
(15 \text { minutes })\end{array}$ & $\begin{array}{r}\text { One-day ahead } \\
(24 \text { hours })\end{array}$ \\
\hline \hline Wind power $P^{w}$ & & \\
Rated power [kW] & 5.0000 & 5.0000 \\
RMSE [kW] & 0.4601 & 0.6654 \\
MAE [kW] & 0.2923 & 0.4425 \\
\hline Solar power $P^{S}$ & & \\
Rated power [kW] & 24.4300 & 24.4300 \\
RMSE [kW] & 1.0753 & 1.4165 \\
MAE [kW] & 0.6949 & 0.8975 \\
\hline Electric load $P^{L}$ & & \\
Max load [kW] & 28.000 & 28.000 \\
RMSE [kW] & 1.6085 & 1.8850 \\
MAE [kW] & 1.1153 & 1.2674 \\
\hline
\end{tabular}

Table I presents the forecasting errors, in terms of RMSE and mean absolute error (MAE) for one-step ahead (15 min) and one-day ahead ( $24 \mathrm{~h}$ ) of the wind power, solar power, and electric load. The prediction errors increase for a larger horizon prediction, as expected. These results are in accordance with the prediction errors shown in Figs. 3, 5, and 7.

Figs. 9-11 show one-day ahead forecast of the fuzzy interval for the wind power, solar power, and electric load, respectively, with $70 \% \mathrm{CP}$, using a rolling horizon strategy, i.e., the forecast for 96 -steps ahead is performed every $15 \mathrm{~min}$. For the wind and solar power, the forecast intervals closely follow the real data. In the electric load case, the intervals are longer due to the small size of the microgrid, implying that each small change in the demand has a significant impact on the load of the microgrid.

\section{F. Comparison With Linear Regression Models}

An analysis of the suitability of the proposed fuzzy prediction interval model, and comparisons with different approaches can be made by assessing its performance on a target application. Thus, the authors are working on examining the performance of the proposed approach when implemented on an EMS for isolated microgrids, based on its ability to provide protection against prediction uncertainty in the microgrid

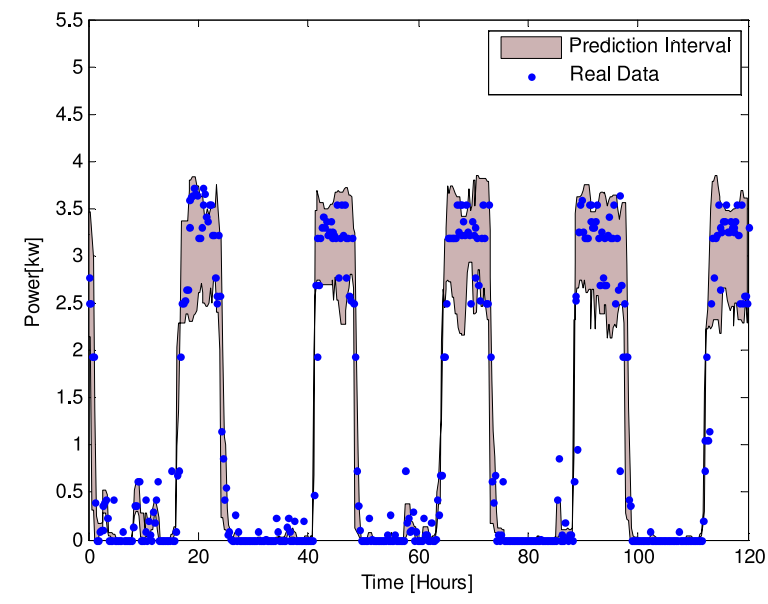

Fig. 9. One-day ahead wind power fuzzy prediction interval using the rolling horizon strategy.

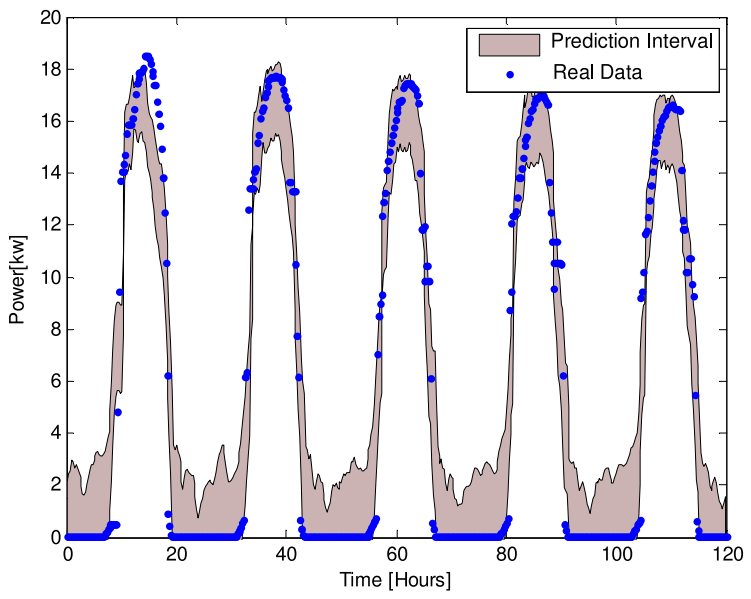

Fig. 10. One-day ahead solar power fuzzy prediction interval using the rolling horizon strategy.

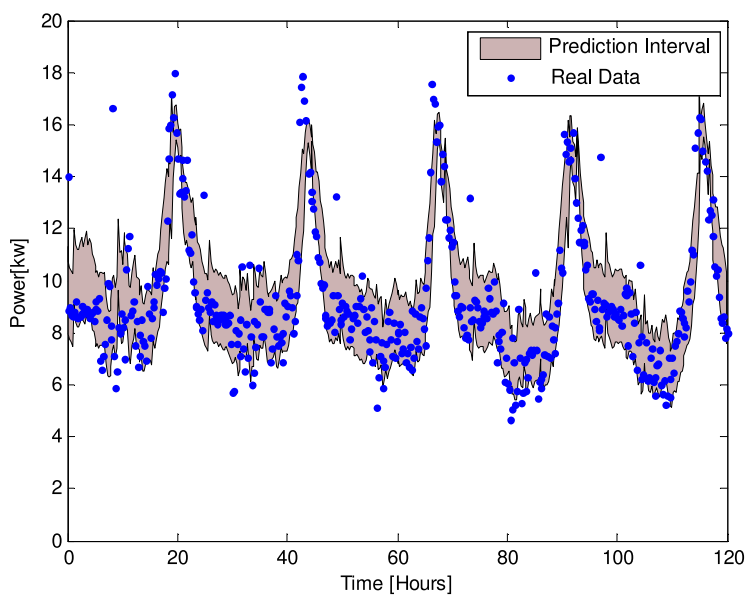

Fig. 11. One-day ahead load fuzzy prediction interval using the rolling horizon strategy.

dispatch, which can be measured in terms of costs of operation and load shedding requirements. Nevertheless, a comparison of the quality of forecasted intervals is presented here based on linear regression models and their associated prediction 
TABLE II

PREDICTION INTERVALS COMPARISON FOR ELECTRIC LOAD

\begin{tabular}{cccc}
\hline \hline Models & $\begin{array}{c}\text { One-step ahead } \\
(15 \text { minutes })\end{array}$ & $\begin{array}{c}\text { One-hour } \\
\text { ahead }\end{array}$ & $\begin{array}{c}\text { One-day ahead } \\
(24 \text { hours })\end{array}$ \\
\hline \hline Linear Regression & & & \\
RMSE [kW] & 1.5291 & 1.8849 & 2.2955 \\
MAE [kW] & 1.0642 & 1.3486 & 1.7159 \\
CP [\%] & 86.83 & 86.93 & 84.99 \\
NAW [\%] & 15.88 & 19.67 & 22.73 \\
\hline Fuzzy Prediction & & & \\
Interval & & & \\
RMSE [kW] & 1.6085 & 1.8782 & 1.8850 \\
MAE [kW] & 1.1153 & 1.2697 & 1.2674 \\
CP [\%] & 88.72 & 88.51 & 87.29 \\
NAW [\%] & 19.07 & 19.63 & 19.08 \\
\hline
\end{tabular}

intervals, for a given $\mathrm{CP}$ of the fuzzy models. Due to their high variability and abundance, load data is used for the comparison.

Table II shows the results of the load prediction intervals for the linear and fuzzy models, with a $90 \% \mathrm{CP}$ of the one-day ahead prediction. The models are compared in terms of RMSE and MAE, whereas the prediction intervals are compared using the CP defined in (23) for the predicted interval, and a normalized average width (NAW) of the predicted interval defined by

$$
\mathrm{NAW}=\frac{1}{M\left[p_{\max }-p_{\min }\right]} \sum_{i=1}^{M}\left[\bar{p}_{i}-\underline{p_{i}}\right]
$$

where $M$ is the size of the data set; $p_{\max }$ and $p_{\min }$ are the maximum and minimum values for the set, respectively; and $\bar{p}_{i}$ and $p_{i}$ are the upper and lower bounds of the prediction interval, respectively. Observe that the fuzzy models are more accurate for one-hour and one-day ahead forecasts in terms of RMSE and MAE indexes, whereas linear regressions outperform the fuzzy models for one-step ahead forecasts. In terms of prediction intervals, fuzzy models provide a higher $\mathrm{CP}$ with narrower intervals for one-day ahead forecasts, and also slightly better intervals for one-hour ahead forecasts. In the case of one-step ahead intervals, even though linear regression models provide a narrower interval, they also yield a lower CP. These results are to be expected given the ability of fuzzy models to better represent the dynamics and nonlinearities of the variables, which are more apparent in longer look-ahead forecasts.

\section{CONCLUSION}

Fuzzy prediction interval models were proposed for microgrids EMS, which facilitate robust predictive control problem. Fuzzy prediction intervals were used to represent the future uncertainty of both nondispatchable power generation and electric load. Fuzzy prediction interval models for the wind power, solar power, and load of a microgrid installed in Chile were generated with different levels of CP using one-day ahead forecasting. Wider intervals were obtained for higher levels of $\mathrm{CP}$, as expected. The solar power interval was the narrowest of the three models generated because solar energy in the studied location does not vary much throughout the year. On the other hand, loads showed wider variations, reflecting the significant impact of load uncertainty in microgrids. The intervals obtained using the proposed method were also compared with respect to those obtained using linear regression models, showing a better performance of the proposed fuzzy models in terms of $\mathrm{CP}$ and interval widths, especially for the case of longer look-ahead forecasts. The worst case obtained from the fuzzy prediction intervals will allow determining the necessary reserves for a microgrid.

Future work will focus on the implementation of a robust EMS using the fuzzy prediction interval models developed herein.

\section{REFERENCES}

[1] F. Bouffard and F. Galiana, "Stochastic security for operations planning with significant wind power generation," IEEE Trans. Power Syst., vol. 23, no. 2, pp. 306-316, May 2008.

[2] A. Papavasiliou, S. Oren, and R. O'Neill, "Reserve requirements for wind power integration: A scenario-based stochastic programming framework," IEEE Trans. Power Syst., vol. 26, no. 4, pp. 2197-2206, Nov. 2011.

[3] J. Ruiwei, W. Jianhui, and G. Yongpei, "Robust unit commitment with wind power and pumped storage hydro," IEEE Trans. Power Syst., vol. 27, no. 2, pp. 800-810, May 2012.

[4] L. Wu, M. Shahidehpour, and T. Li, "Stochastic security-constrained unit commitment," IEEE Trans. Power Syst., vol. 22, no. 2, pp. 800-811, May 2007.

[5] F. Xu, M. Leung, and C. Zhou, "A RBF network for short-term load forecast on microgrid," in Proc. Int. Conf. Mach. Learn. Cybern., vol. 6. Qingdao, China, Jul. 2010, pp. 3195-3199.

[6] P. Chan, W. Chen, W. Ng, and D. Yeung, "Multiple classifier system for short term load forecast of microgrid," in Proc. Int. Conf. Mach. Learn. Cybern., vol. 3. Guilin, China, Jul. 2011, pp. 1268-1273.

[7] R. Palma-Behnke, C. Benavides, E. Aranda, J. Llanos, and D. Sáez, "Energy management system for a renewable based micro-grid with a demand side management mechanism," in Proc. IEEE Symp. Ser Comput. Intell. Appl. Smart Grid, Paris, France, Apr. 2011, pp. 1-8.

[8] Z. Bashir and M. El-Hawary, "Applying wavelets to short-term load forecasting using PSO-based neural networks," IEEE Trans. Power Syst., vol. 24, no. 1, pp. 20-27, Feb. 2009.

[9] W. Hinojosa and A. Hoese, "Short-term forecasting using fuzzy inductive reasoning evolutionary algorithms," IEEE Trans. Power Syst., vol. 25, no. 1, pp. 565-574, Feb. 2010.

[10] N. Amjady, F. Keynia, and H. Zareipour, "Short-term load forecast of micro-grids by a new bilevel prediction strategy," IEEE Trans. Smart Grid, vol. 1, no. 3, pp. 286-294, Dec. 2010.

[11] P. McSharry, S. Bouwman, and G. Bloemhof, "Probabilistic forecasts of the magnitude and timing of peak electricity demand," IEEE Trans. Power Syst., vol. 20, no. 2, pp. 1166-1172, May 2005.

[12] R. Hyndman and S. Fan, "Density forecasting for long-term peak electricity demand," IEEE Trans. Power Syst., vol. 25, no. 2, pp. 1142-1153, May 2010.

[13] T. Hong, J. Wilson, and J. Xie, "Long term probabilistic load forecasting and normalization with hourly information," IEEE Trans. Smart Grid, vol. 5, no. 1, pp. 456-462, Jan. 2014.

[14] R. Kavasseri and K. Seetharaman, "Day-ahead wind speed forecasting using f-ARIMA models," Renew. Energy, vol. 34, no. 5, pp. 1388-1393, 2009.

[15] F. Deng, G. Su, C. Liu, and Z. Wang, "Global solar radiation modeling using the artificial neural network technique," in Proc. Asia-Pac. Power Energy Eng. Conf., Chengdu, China, Mar. 2010, pp. 1-5.

[16] S. Kélouwani and K. Agbossou, "Nonlinear model identification of wind turbine with a neural network," IEEE Trans. Energy Convers., vol. 19, no. 3, pp. 607-612, Sep. 2004.

[17] A. Chatterjee and A. Keyhani, "Neural network estimation of microgrid maximum solar power," IEEE Trans. Smart Grid, vol. 3, no. 4, pp. 1860-1866, Dec. 2012.

[18] T. Hong and P. Wang, "Fuzzy interaction regression for short term load forecasting," Fuzzy Optim. Decis. Mak., vol. 13, no. 1, pp. 91-103, 2014.

[19] D. Olivares, C. Cañizares, and M. Kazerani, "A centralized optimal energy management system for microgrids," in Proc. IEEE Power Energy Soc. Gen. Meeting, San Diego, CA, USA, Jul. 2011, pp. 1-6. 
[20] R. Palma-Behnke et al., "A microgrid energy management system based on the rolling horizon strategy," IEEE Trans. Smart Grid, vol. 4, no. 2, pp. 996-1006, Jun. 2013.

[21] A. Tsikalakis and N. Hatziargyriou, "Centralized control for optimizing micro-grids operation," IEEE Trans. Energy Convers., vol. 23, no. 1, pp. 241-248, Mar. 2008.

[22] H. Morais, P. Kádar, P. Faria, Z. Vale, and H. Khodr, "Optimal scheduling of a renewable micro-grid in an isolated load area using mixed-integer linear programming," Renew. Energy, vol. 35, no. 1, pp. 151-156, Jan. 2010.

[23] I. Škrjanc, "Fuzzy confidence interval for pH titration curve," Appl. Math. Model., vol. 35, no. 8, pp. 4083-4090, Aug. 2011.

[24] A. Núñez and B. De Schutter, "Distributed fuzzy confidence interval for traffic measurements," in Proc. 51st IEEE Conf. Decis. Control, Maui, HI, USA, Dec. 2012, pp. 6995-7000.

[25] T. Takagi and M. Sugeno, "Fuzzy identification of systems and its applications to modeling and control," IEEE Trans. Syst., Man, Cybern., vol. 15, no. 1, pp. 116-132, Jan./Feb. 2005.

[26] M. Sugeno and T. Yasukawa, "A fuzzy logic based approach to qualitative modeling," IEEE Trans. Fuzzy Syst., vol. 1, no. 1, pp. 7-31, Feb. 1993.

[27] R. Babuska, Fuzzy Modelling for Control. Boston, MA, USA: Kluwer, 1998.

[28] J. Llanos, D. Sáez, R. Palma-Behnke, A. Nuñez, and G. Jiménez-Estevez, "Load profile generator and load forecasting for a renewable based microgrid using self organizing maps and neural networks," in Proc. Int. Joint Conf. Neural Netw., Brisbane, QLD, Australia, 2012, pp. 1-8.

Doris Sáez (M'94-SM'06) received the M.Sc. and Ph.D. degrees in electrical engineering from Pontificia Universidad Católica de Chile, Santiago, Chile, in 1995 and 2000, respectively.

She is currently an Associate Professor with the Department of Electrical Engineering, University of Chile, Santiago. Her current research interests include predictive control, fuzzy control design, fuzzy identification, control of power generation plants, and control of transport systems. She has coauthored the books entitled Hybrid Predictive Control for Dynamic Transport Problems (Springer-Verlag, 2013) and Optimization of Industrial Processes at Supervisory Level: Application to Control of Thermal Power Plants (Springer-Verlag, 2002).

Dr. Sáez is an Associate Editor of the IEEE TRAnSACTIONS ON FUZZY SYSTEMS and Soft Computing.

Fernanda Ávila received the B.Sc. and M.Sc. degrees in electrical engineering from the University of Chile, Santiago, Chile, in 2011 and 2013, respectively.

Her current research interests include fuzzy systems and renewable energy plants.
Daniel Olivares (S'11-M'14) received the B.Sc. and Engineering degrees in electrical engineering from the University of Chile, Santiago, Chile, in 2006 and 2008, respectively, and the Ph.D. degree in electrical and computer engineering from the University of Waterloo, Waterloo, ON, Canada, in 2014.

$\mathrm{He}$ is currently an Assistant Professor with the Electrical Engineering Department, Pontificia Universidad Católica de Chile, Santiago. His current research interests include the modeling, simulation, control, and optimization of power systems in the context of smart grids.

Claudio Cañizares (S'86-M'91-SM'00-F'07) received the Diploma degree in electrical engineering from Escuela Politecnica Nacional, Quito, Ecuador, in 1984, and the M.S. and Ph.D. degrees in electrical engineering from the University of Wisconsin, Madison, WI, USA, in 1988 and 1991, respectively.

$\mathrm{He}$ is currently a Full Professor with the Electrical and Computer Engineering Department, University of Waterloo, Waterloo, ON, Canada, and an Associate Director of the Waterloo Institute for Sustainable Energy, Waterloo. His current research interests include the study of stability, modeling, simulation, control, and computational issues in power systems within the context of competitive electricity markets and smart grids.

Dr. Cañizares was the recipient of the Hydro One Research Chair in 2010 and various IEEE-Power and Energy Society (PES) Working Group awards, and has held several leadership appointments with IEEE-PES technical committees and subcommittees. He is a Registered Professional Engineer in the province of Ontario and a Fellow of the Royal Society of Canada.

Luis Marín received the degree in electronic engineering from the University of Autónoma de Colombia, Bogotá, Colombia, and the M.Sc. degree in information sciences and communications from the Universidad Distrital Francisco José de Caldas, Bogotá, in 2003 and 2012, respectively. He is currently pursuing the $\mathrm{Ph} . \mathrm{D}$. degree in electrical engineering from the University of Chile, Santiago, Chile.

His current research interests include predictive control, renewable energy plants, and robust control for microgrids. 